\title{
Impact of COVID-19 lockdown on adherence to continuous positive airway pressure by obstructive sleep apnoea patients
}

\author{
To the Editor:
}

The psychological effects of the coronavirus 2019 (COVID-19) lockdown and fear of aerosolisation made us think that adherence to continuous positive airway pressure (CPAP) treatment might be reduced and consequently the morbidity and mortality of vulnerable obstructive sleep apnoea (OSA) patients increased.

We analysed the impact of the COVID-19 national lockdown in France on objective adherence to CPAP assessed by telemonitoring in a cohort of 7485 patients with OSA between 15 January 2019 and 15 April 2020 [1].

Comparing data from the pre-COVID-19 period (1 month before 15 March 2020, the date of the national lockdown announcement) to data post-lockdown, there was a 3.9\% $(\mathrm{p}<0.001)$ increase in adherence from a mean value of $386 \mathrm{~min}$ per night pre-COVID-19 to $401 \mathrm{~min}$ per night during lockdown. These data were confirmed by comparing the CPAP adherence rate during the first month of lockdown (15 March 2020 to 15 April 2020) to CPAP adherence during the same time-period in 2019 , with a $4.48 \%$ increase during lockdown $(\mathrm{p}<0.001)$. The proportion of very low adherers $(<10 \mathrm{~min}$ of CPAP use per night $)$ dropped by $18.5 \%(\mathrm{p}<0.001)$ between the similar periods in 2019 and 2020.

These are the first data from a large prospective cohort of patients treated by CPAP, confirming a significant improvement in CPAP adherence during the COVID-19 lockdown. CPAP efficacy is often limited by nonadherence related to a lack of disease-related risk perception [2]. In our case, massive communication about COVID-19, described as a threat to the upper and lower airways, might have motivated patients to be more adherent so as to protect their airways and themselves from the disease, particularly poor adherents. Other hypotheses are that most patients with OSA also have cardiovascular risk factors or comorbidities found to be highly prevalent in coronavirus-infected patients, particularly in patients admitted to an intensive care unit or having the worst prognosis [3]. Moreover, the fear of being hospitalised could have motivated patients to increase CPAP adherence. Finally, staying at home continuously reduced air pollution and or the absence of occupational stress may also have enhanced CPAP use by increasing sleep duration and favouring naps. While a reduction in CPAP adherence has been widely reported in various settings (e.g. post-traumatic stress disorders, sleep disorders) or in clinical trials, a sudden sharp increase (within 1 month) as seen in this national cohort has never been reported [4]. However, the clinical impact of this modest increase in CPAP adherence needs to be confirmed in a larger cohort with outcome data.

Our observation suggests that behavioural interventions, based on the patients' perception of both disease-related risk and CPAP-related benefits could improve adherence. Finally, the abundance of data obtained by CPAP tracking systems (particularly adherence) could be used beyond disease management to monitor behaviour and the impact of diseases like COVID-19, so as to rapidly identify new outbreaks and enable prompt intervention to avoid further complications of the disease and its propagation.

@ERSpublications

In a large prospective cohort of sleep apnoea patients treated by CPAP, adherence to CPAP was significantly increased during the COVID-19 lockdown. This was associated with a shift of patients considered as low adherers to average or high adherers. https://bit.ly/3cBBOhs

Cite this article as: Attias D, Pepin JL, Pathak A. Impact of COVID-19 lockdown on adherence to continuous positive airway pressure by obstructive sleep apnoea patients. Eur Respir J 2020; 56: 2001607 [https://doi.org/10.1183/13993003.01607-2020]. 


\section{David Attias ${ }^{1}$, Jean Louis Pepin ${ }^{2}$ and Atul Pathak ${ }^{3}$}

${ }^{1}$ Dept of Pulmonary and Sleep Medicine, Clinique Pasteur, Toulouse, France. ${ }^{2}$ HP2 Laboratory, INSERM U1042, Grenoble Alpes University, Grenoble, France. ${ }^{3}$ Dept of Cardiovascular Medicine, Princess Grace Hospital, Monaco, Monaco.

Correspondence: Atul Pathak, Dept of Cardiovascular Medicine, Princess Grace Hospital Centre, 1 Avenue Pasteur, Monaco 98012, Monaco, France. E-mail: atul.pathak@chpg.mc

Received: 5 May 2020 | Accepted after revision: 10 May 2020

Conflict of interest: D. Attias has nothing to disclose J.L. Pépin reports grants, personal fees and other from Air Liquide Foundation, Agiradom, AstraZeneca, Philips and Resmed, grants and other from Fisher and Paykel, Mutualia and Vitalaire, personal fees from Boehringer Ingelheim, Jazz Pharmaceutical, Night Balance and Sefam, outside the submitted work. A. Pathak has nothing to disclose.

\section{References}

1 Cistulli PA, Armitstead J, Pepin JL, et al. Short-term CPAP adherence in obstructive sleep apnea: a big data analysis using real world data. Sleep Med 2019; 59: 114-116.

2 Weaver TE. Novel aspects of CPAP treatment and interventions to improve CPAP adherence. J Clin Med 2019; 8: E2220.

3 Wang $\mathrm{D}, \mathrm{Hu} \mathrm{B}, \mathrm{Hu}$ C, et al. Clinical characteristics of 138 hospitalized patients with 2019 novel coronavirusinfected pneumonia in Wuhan, China. JAMA 2020; 323: 1061-1069.

4 Collen JF, Lettieri CJ, Hoffman M. The impact of posttraumatic stress disorder on CPAP adherence in patients with obstructive sleep apnea. J Clin Sleep Med 2012; 8: 667-672. 